\title{
Ultra-Wideband Circuits, Systems, and Applications
}

\author{
Yo-Sheng Lin, ${ }^{1}$ Baoyong Chi, ${ }^{2}$ Hsien-Chin Chiu, ${ }^{3}$ and Hsiao-Chin Chen ${ }^{4}$ \\ ${ }^{1}$ Department of Electrical Engineering, National Chi Nan University, Puli 545, Nantou, Taiwan \\ ${ }^{2}$ Institute of Microelectronics, Tsinghua University, Beijing 100084, China \\ ${ }^{3}$ Department of Electronic Engineering, Chang Gung University, Kwei-Shan 333, Taoyuan, Taiwan \\ ${ }^{4}$ Department of Electrical Engineering, National Taiwan University of Science and Technology, Taipei 106, Taiwan
}

Correspondence should be addressed to Yo-Sheng Lin, stephenlin@ncnu.edu.tw

Received 21 May 2012; Accepted 21 May 2012

Copyright (C) 2012 Yo-Sheng Lin et al. This is an open access article distributed under the Creative Commons Attribution License, which permits unrestricted use, distribution, and reproduction in any medium, provided the original work is properly cited.

Ultra-wideband (UWB) technology include many applications, such as WiGig, home networking, wireless universal serial bus (USB), wireless personal area network (WPAN), wireless body area network (WBAN), healthcare and medical imaging, and automotive radar. The burgeoning application of UWB technology has brought about new challenges and opportunities for both the academia and the industry. For example, recently, many groups are dedicated to the application of UWB technology on medical sensing, localization, and communication, which leads to potential applications in medicine, especially for less invasive medical diagnosis and monitoring. Undoubtedly, with UWB technology, current wireless health systems and novel medical applications can be further improved and developed.

Ka-band UWB vehicular radars can inherently achieve high-range resolution. In the research article entitled "Performance analysis of ultra-wideband channel for shortrange monopulse radar at Ka-band," the development and measurement results of a prototype UWB monopulse radar equipped with a two-element receiving antenna array are illustrated. Additionally, to design suitable radar's waveforms, performance degradation attributed to a number of averaged received monopulses is examined.

Furthermore, in the research article entitled "Analysis and mitigation of the narrowband interference impact on IRUWB communication systems," the impact of narrowband interference signals on impulse radio UWB communication systems is investigated by proposing an interference canceller scheme. This scheme is capable of suppressing the impact of such interference and enhancing the performance of UWB communication systems.
UWB signal generation is critical in UWB communication systems. In the research article entitled "All-optical fiberinterferometer-based methods for ultra-wideband signal generation," two new, simple, and cost-effective all-optical methods for generating UWB impulse radio signals are reported. These methods not only generate UWB pulses optically but also assure the propagation over optical networks.

UWB signals show robustness against multipath interference and allow for high-accuracy positioning. Thus, it is promising to apply them in real-time locating systems (RTLSs) and wireless sensor networks which adopt the IEEE 802.15.4a standard. In the research article entitled "Ranging performance of the IEEE 802.15.4a UWB standard under FCC/CEPT regulations," a coherent receiver and an energy detector (i.e., a noncoherent receiver) are studied for ranging in IEEE 802.15.4a, in the sense of maximal allowed transmit energy and path-loss, and maximal operating distance.

In the research article entitled "UWB localization system for indoor applications: concept, realization and analysis," a complete UWB indoor localization demonstrator is presented. This demonstrator is targeted on operation with a predeployed access point infrastructure. The proposed methods have improved the average accuracy from $9 \mathrm{~cm}$ to $2.5 \mathrm{~cm}$.

The inherent features of the UWB radio signals make them highly suitable for less invasive medical application. For example, the UWB radar may be used in novel noninvasive sensing and imaging techniques thanks to its high temporal resolution for detecting backscattered signals. In the review article entitled "Ultra-wideband technology in medicine: a survey," the authors described their current research on the application of the UWB technology to noninvasive 
measurement of blood pressure. In addition, they reported a survey of recent research in UWB technology for medical sensing and communications.

The Guest Editors of this special issue acknowledge all the authors who responded to the call for papers, and they thank all the reviewers for their dedication for ensuring a high standard for the selected papers. Though this special issue only covers some but not all of the important areas related to ultra-wideband circuits, systems, and applications, we hope that readers will find this issue insightful and useful.

Yo-Sheng Lin

Baoyong Chi

Hsien-Chin Chiu

Hsiao-Chin Chen 

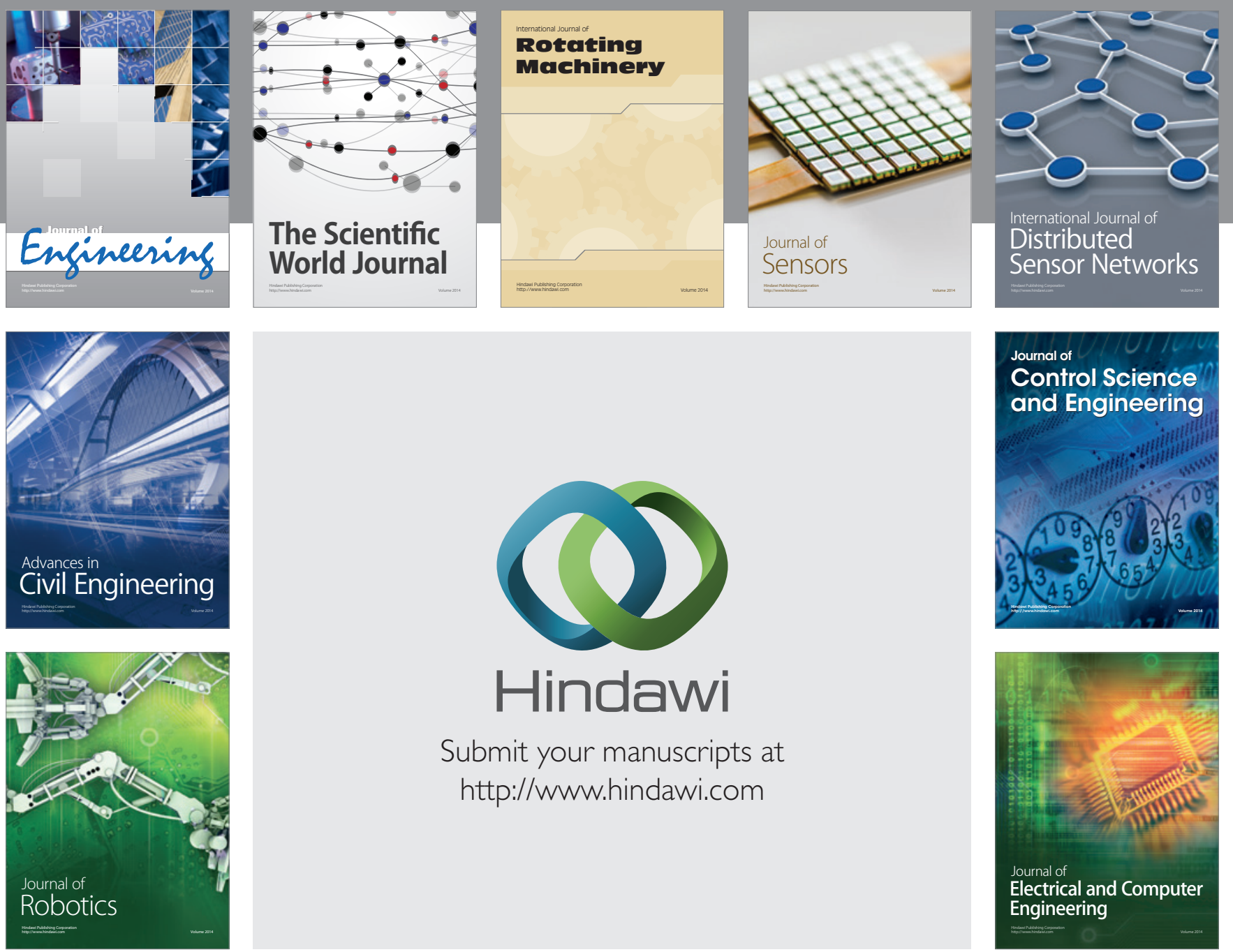

Submit your manuscripts at

http://www.hindawi.com
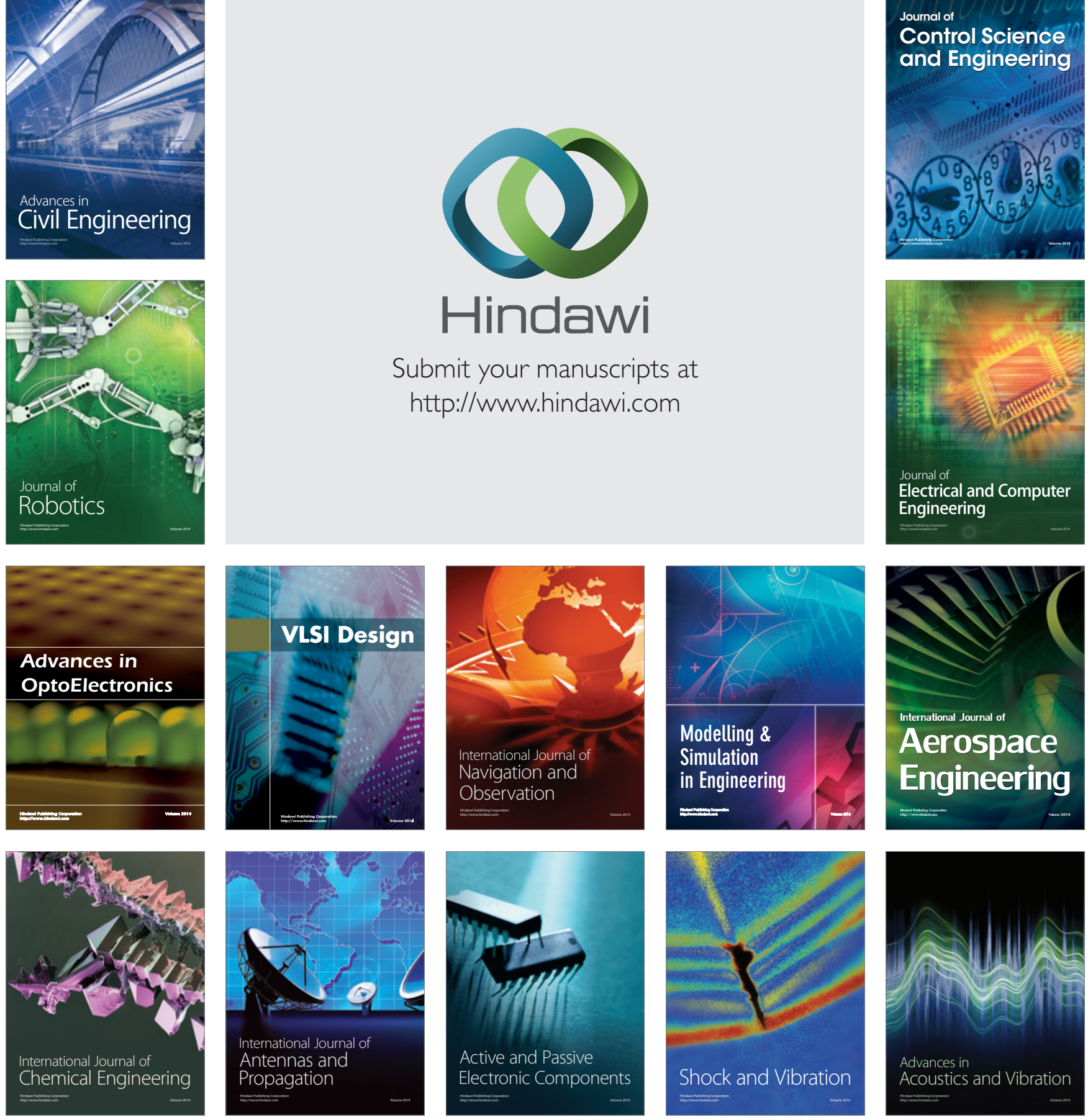